\title{
Oscillating glucose induces microRNA-185 and impairs an efficient antioxidant response in human endothelial cells
}

\author{
Lucia La Sala ${ }^{1}$, Monica Cattaneo ${ }^{1}$, Valeria De Nigris², Gemma Pujadas², Roberto Testa ${ }^{3}$, Anna R. Bonfigli ${ }^{4}$, \\ Stefano Genovese ${ }^{1}$ and Antonio Ceriello ${ }^{2^{*}}$
}

\begin{abstract}
Background: Intracellular antioxidant response to high glucose is mediated by $\mathrm{Cu} / \mathrm{Mn}$-superoxide dismutases (SOD-1/SOD-2), catalase (CAT) and glutathione peroxidases (GPx), particularly glutathione peroxidase-1 (GPx-1). Although oscillating glucose can induce a more deleterious effect than high glucose on endothelial cells, the mechanism by which oscillating glucose exerts its dangerous effects is incompletely understood; however, the involvement of oxidative damage has been generally accepted. In this study we sought to determine whether oscillating glucose differentially modulates antioxidant response, and to elucidate the potential regulatory mechanisms exerted by the microRNA-185 (miR-185).
\end{abstract}

Methods: Human endothelial cells were exposed for 1 week to constant and oscillating high glucose. SOD-1, SOD-2, CAT and GPx-1, as well as two markers of oxidative stress [8-hydroxy-2'-deoxyguanosine (8-OHdG) and the phosphorylated form of $\mathrm{H} 2 \mathrm{AX}(\mathrm{Y}-\mathrm{H} 2 \mathrm{AX})$ ] were measured at the end of the experiment. Intracellular miR-185 was measured and loss-of function assays were performed in HUVEC. Bioinformatic tool was used to predict the link between miR-185 on $3^{\prime}$ UTR of GPX-1 gene. Luciferase assay was performed to confirm the binding on HUVEC.

Results: After exposure to constant high glucose SOD-1 and GPX-1 increased, while in oscillating glucose SOD-1 increased and GPx-1 did not. SOD-2 and CAT remained unchanged under both conditions. A critical involvement of oscillating glucose-induced miR-185 in the dysregulation of endogenous GPx-1 was found. Computational analyses predict GPX-1 as miR-185's target. HUVEC cultures were used to confirm glucose's causal role on the expression of miR-185, its target mRNA and protein and finally the activation of antioxidant response. In vitro luciferase assays confirmed computational predictions targeting of miR-185 on 3'-UTR of GPx-1 mRNA. Knockdown of miR-185, using anti-miR-185 inhibitor, was accompanied by a significant upregulation of GPx-1 in oscillating glucose. 8-OHdG and $\mathrm{Y}$-H2AX increased more in oscillating glucose than in constant high glucose.

Conclusions: Glucose oscillations may exert more deleterious effects on the endothelium than high glucose, likely due to an impaired response of GPx-1, coupled by the upregulation of miR-185.

Keywords: Oscillating glucose, miR-185, Antioxidant defense, GPx-1, Oxidative stress

\section{Background}

It has recently been suggested that glucose variability may be an independent risk factor for vascular complications

\footnotetext{
*Correspondence: aceriell@clinic.ub.es

${ }^{2}$ Insititut d'Investigacions Biomèdiques August Pi i Sunyer (IDIBAPS) and Centro de Investigación Biomedica en Red de Diabetes y Enfermedades Metabólicas Asociadas (CIBERDEM), Barcelona, Spain

Full list of author information is available at the end of the article
}

of diabetes [1, 2]. Recently, glucose oscillations, like those experienced daily by diabetic patients, have been demonstrated to be more dangerous in vitro than constant high glucose; this is true for several cell types, including endothelial cells [3-7]. A higher generation of free radicals during glucose oscillations has been hypothesized as a causal factor for this phenomenon [3-7]. 
It has been shown that endogenous antioxidant enzymes protect cells against the toxic effect of reactive oxygen species (ROS) and are an essential defence system against oxidative injury [8]. Under normal physiological conditions, ROS production is balanced by an efficient system of antioxidants: such molecules are capable of neutralizing them and thereby preventing oxidant damage. Superoxide anion, a highly reactive molecule, can be converted into less reactive hydrogen peroxide $\left(\mathrm{H}_{2} \mathrm{O}_{2}\right)$ by the cytosolic $\mathrm{Cu} / \mathrm{Zn}$-superoxide dismutase (SOD-1), and by the mitochondrial located $\mathrm{Mn}$-superoxide dismutase (SOD-2), whereas glutathione peroxidase-1 (GPx-1) and catalase (CAT) play a role in the further enzymatic catabolism of ROS [8].

The aim of this study was to compare the response in human endothelial cells of this antioxidant system during glucose oscillation to that which takes place during chronic stable glucose exposure.

A new class of small non-coding RNAs, termed microRNAs (miRNAs or miRs), is emerging as new regulators of metabolism during development and disease [9]. miRNAs are endogenous $\sim 23$ nt long, non-coding RNA molecules that play important gene regulatory roles in cells by pairing to the un-translated region (3'-UTR) of mRNAs of protein-coding genes in order to direct their post-transcriptional repression [10]. miRNA recognises complementary miRNA recognition elements (MRE) throughout mRNA sequences, including $3^{\prime}$ - and $5^{\prime}$-UTRs. The dysregulation of miRNA expression can affect the expression of hundreds mRNAs and proteins. miRNAs are found in different genomic regions: introns of protein-coding genes; exons and introns of non-coding genes and even the $3^{\prime}$-untranslated region $\left(3^{\prime}\right.$-UTR) of protein-coding genes [12].

Multiple studies have demonstrated that a large number of miRNAs is under the control of various metabolic stimuli, including glucose [11]. Although many miRNAs have already been identified, their roles in the regulation of key genes and signaling pathways associated with glucose stimuli still remain poorly understood. Recently, microRNA-185 (miR-185) has been related to altered expression of selenoproteins, including altered GPx-1 [13], but its direct binding did have not yet been reported. Therefore, in this work, we sought to evaluate the possible modulation carried out by miR-185 during glucose oscillations on GPx-1 expression.

\section{Methods}

\section{Materials and cell cultures}

Primary pooled human umbilical vein endothelial cells (HUVECs) and growth factors were purchased from Lonza (Lonza Bioresearch LBS, Basel, Switzerland). Cells were maintained for 3 weeks in endothelial basal medium, supplemented with low fetal bovine serum (2 \%), hydrocortisone $(1 \mu \mathrm{g} / \mathrm{mL})$, basal fibroblastic growth factor $(5 \mathrm{ng} / \mathrm{mL})$, epidermal growth factor $(5 \mathrm{ng} /$ $\mathrm{mL})$, heparin $(0.75$ units $/ \mathrm{mL})$ and gentamicin/amphotericin (GA-1000, $0.1 \%$ ) in a humidified incubator with $5 \%$ carbon dioxide added.

\section{Experimental design and glucose exposures}

$2 \times 10^{5} \mathrm{HUVECs} /$ well were seeded in 6-well plates (Corning, NY, USA) and exposed for 7 days to three different glucose concentrations: normal glucose (NG; $5 \mathrm{mmol} / \mathrm{l}$ ), oscillating glucose (OG; $5 / 25 \mathrm{mmol} / \mathrm{l}$ ) and high glucose (HG; $25 \mathrm{mmol} / \mathrm{l})$. OG condition was obtained changing glucose concentration (from 5 to $25 \mathrm{mmol} / \mathrm{l}$ ) every day. Experimental control was performed incubating the cells with mannitol at the same concentration of glucose.

\section{Determination of 8-hydroxy-2'-deoxyguanosine (8-OHdG)}

8-OH-dG, a marker of oxidative stress, was determined in the HUVECs using Bioxytech 8-OHdG-EIA Kit (OXIS Health Products, Portland, OR, USA).

\section{RNA extraction and Real-time PCR (q-PCR) analysis}

Total RNA was extracted using an RNA purification kit (NorgenBiotek, Thorold, ON, Canada). One microgram of total RNA was reverse transcribed using the SuperScript III reverse transcriptase and random hexamers (Invitrogen, Life Technologies, Grand Island, NY, USA). q-PCR was performed using the ABI 7900 HT thermocycler (Applied Biosystems, Life Technologies, Grand Island, NY, USA), in a reaction buffer using Taqman Gene expression Master Mix, with pre-optimized primers and probes obtained from Applied Biosystems, and using SYBR green ready mix $\left(\mathrm{SYBR}^{\circledR}{ }^{\circledR}\right.$ Premix Ex $\mathrm{Taq}^{\mathrm{TM}}$ II, Taqara, Japan). The list of primers used is reported in Table 1. All $q-P C R$ were normalized to actin-beta, as a housekeeping gene.

\section{Endogenous expression, mimic and inhibition of miR-185} miR-185 expression was examined with the TaqMan MicroRNA Assay Kit (Applied Biosystems, Life Technologies, Grand Island, NY, USA). MultiScribe

\section{Table 1 List of primers}

\begin{tabular}{lll}
\hline Gene & Sequence & Accession number \\
\hline GPX-1 & 5'-CCCAGTCGGTGTATGCCTTC-3' & NM_000581.2 \\
SOD-2 & 5'-AGCATGAAGTTGGGCTCGAA-3' & \\
& 5'-GGCCTACGTGAACAACCTGA-3' & NM_001024465 \\
5'-CAGGACGTTATCTTGCTGGG-3' & \\
SOD-1 & Hs00533490_m1 & NM_000454.4 \\
CAT & Hs00156308_m1 & NM_001752.3 \\
ACTB & Hs99999903_m1 & NM_001101.3 \\
\hline
\end{tabular}


Reverse Transcriptase was used for RT-PCR, and TaqMan primers for hsa-miR-185 (assay ID 002271) were used to monitor miR-185 expression. RNU44/48 or SNORD-44/48 (assay ID 001094/ID 001006) was used as endogenous miRNA controls (all purchased from Applied Biosystems). has-miR-185-5p mirVana ${ }^{\circledR}$ miRNA mimic (MC12486), Anti-miR ${ }^{\mathrm{TM}}$ miRNA-185 inhibitor (AM12486), an antisense miR-185, and scrambled Anti$\mathrm{miR}^{\mathrm{TM}}$ miRNA inhibitor negative control (AM17010) was purchased from Ambion (Foster City, CA, USA). Transfections of miRNA-185 inhibitors were performed at least three times in triplicate using INTERFERin ${ }^{\circledR}$ transfection reagent according to the manufacturer's protocol (POLYPLUS-transfection, NY, USA).

\section{Target predictions of miRNAs}

The target gene predictions of human miRNAs have been gathered from a publicly available database for miRNAs target predictions (TargetScan 5.2, http://www.targetscan.org, for poorly conserved sites [14]). Sequence for miRNA was obtained from the miRNA database, miRBase (Faculty of Life Sciences, University of Manchester). RNA hybrid tool [15] was used to predict the resulting secondary structure formed by interacting mRNA and miRNA and calculate $\Delta G$ minimum free energy. RNA hybrid is available at https://bibiserv.techfak.unibiekefeld.de/rnahybrid.

\section{HUVEC co-transfection for functional assay}

HUVEC $5 \times 10^{4}$ cells passage $4(\mathrm{p} 4)$ were transiently cotransfected with $3^{\prime}$-UTR-GPx-1 expression vector firefly luciferase reporter assay (Origene, MD, USA), containing the entire 217 bp GPx-1 3'-UTR together with miRNA185 mimic sequence, using jetPRIME co-transfection reagent following manufacturer's instructions (POLYPLUS). As controls, cells were transfected with empty vector (pMIR) alone, and with pMIR with miRNA-185 mimic sequence. Cells were processed for lysis and collected $72 \mathrm{~h}$ after co-transfection and luciferase activity of total cell lysates measured using an established luciferase reporter assay kit (Dual Luciferase Reporter System, Promega, USA). Luciferase values were normalized calculating $\mathrm{RLU} / \mu \mathrm{g}$ protein.

\section{Western immunoblots}

Whole cell lysates were prepared using RIPA buffer (Sigma-Aldrich, St. Louis, MO, USA) containing a protease and phosphatase inhibitor cocktail. Protein contents were determined using the Bradford Reagent (Sigma-Aldrich, St. Louis, MO, USA). Whole cell lysates and chromatin-bound nuclear extracts were subjected to 4-20\% Tris-glycine gradient (SDS-PAGE) gels
(Lonza Bioresearch LBS, Basel, Switzerland) in reducing conditions and blotted onto a polyvilynidene fluoride membrane. After blocking with $5 \%$ non-fat dry milk in $20 \mathrm{mM}$ Tris- $\mathrm{HCl}$ (pH 7.5), $135 \mathrm{mM} \mathrm{NaCl}$, and $0.1 \%$ Tween-20, blots were incubated with polyclonal antibodies against phospho-histone2AX $(\gamma-\mathrm{H} 2 \mathrm{AX})$ obtained from Cell Signaling (Beverly, MA, USA), washed with $20 \mathrm{mM}$ Tris-HCl (pH 7.5), $135 \mathrm{mM} \mathrm{NaCl}$, and $0.1 \%$ Tween-20, and incubated with a horseradish peroxidaseconjugated secondary antibody. Proteins were detected using the ECL system (Pierce Chemical, Rockford, IL, USA), according to the manufacturer's instructions and revealed using a CCD camera (ImageQuantLAS4000, GE Healthcare, UK). Antibodies against SOD-1, CAT, GPx-1 (Cell Signaling, Beverly, MA, USA), and SOD-2 (Santa Cruz Biotechnology, CA, USA) were blotted in the same conditions. $\beta$-Actin (Sigma-Aldrich, St. Louis, MO, USA) was used as loaded control, whereas histoneH3 (Abcam) was used for a chromatin-bound nuclear extract loaded control. Protein content quantification was performed using computer-assisted densitometry (http://www.imagej.nih.gov, ImageJ, NIH, Bethesda, MD, USA).

\section{Subcellular protein extracts}

Chromatin-bound nuclear extracts were prepared using Subcellular Protein Fractionation Kit for Cultured Cells (Pierce Chemical, Rockford, IL, USA) according to the manufacturer's description. Thus, HUVECs $\left(2 \times 10^{6}\right)$ cells were fractionated, equal amounts of chromatinbound nuclear extracts $(30 \mu \mathrm{g})$ were separated in 4-20\% Tris-glycine gradient gels (Lonza Bioresearch LBS, Basel, Switzerland), and then run on SDS-PAGE in reducing conditions.

\section{Measurement of GPx activity}

HUVECs $\left(1 \times 10^{6}\right)$ were subjected to lysis and sonication $(2 \times 30 \mathrm{~s})$ on ice. Supernatants were collected and assayed for protein content using Bradford assay. GPx protein activity was measured by GPx activity colorimetric assay kit (Biovision, Milpitas, CA, USA) according to the manufacturer's instructions and normalized by protein content.

\section{Statistical analysis}

Results are expressed as mean \pm SEM. Statistical analysis was performed using GraphPrism5.0 ${ }^{\circledR}$ (http://www. graphpad.com). Differences between groups were carried out by one-way ANOVA, followed by the Tukey's post hoc test. Significant differences were assumed at $\mathrm{p}<0.05$. Three different experiments were performed in triplicate to ensure reproducibility. 


\section{Results}

GPx-1 response is altered in oscillating glucose

The endogenous expression of SOD-1, SOD-2, CAT and GPx-1 were assessed with $q$-PCR and western blot analysis (Fig. 1a-c). In HG in respect to NG, SOD-1 [mRNA $(\mathrm{p}<0.01)$ and protein $(\mathrm{p}<0.01)$ ] and GPx-1 [mRNA $(\mathrm{p}<0.05)$ and protein $(\mathrm{p}<0.01)]$ were increased (Fig. 1ac). In OG in respect to NG, SOD-1 [mRNA $(\mathrm{p}<0.05)$ and protein $(\mathrm{p}<0.05)$ ] was increased, while GPx-1 (mRNA and protein) did not change (Fig. 1a-c). This profile of GPx-1 was also confirmed for protein activity (Fig. 1d). After exposure to HG, GPx activity was significantly higher vs NG and OG $(\mathrm{p}<0.001)$. GPx activity was not different in OG vs NG ( $\mathrm{p}=\mathrm{ns})$. SOD-2 and CAT were unchanged in both conditions, even though there was a tendency for CAT to increase ( $\mathrm{p}=0.09)$, (Fig. 1a-c).
Effects of $\mathrm{OG}$ and $\mathrm{HG}$ on oxidative stress markers

The 8-OHdG content and $\gamma$-H2AX levels in HUVECs, as markers of oxidative damage to nucleic acids, were investigated. Our results shown increased 8-OHdG in both OG ( $\mathrm{p}<0.001)$ and HG $(\mathrm{p}<0.01)$ compared to NG (Fig. 2a). However, OG induced more damage on DNA (8-OHdG) than HG (OG vs HG, p < 0.05). Similarly, HG $(\mathrm{p}<0.05)$ and OG $(\mathrm{p}<0.001)$ promoted increased double strand breaks in the cells, demonstrated by $\gamma-\mathrm{H} 2 \mathrm{AX}$ formation (Fig. 2b). OG induced more $\gamma$-H2AX than HG (OG vs HG, $\mathrm{p}<0.001$ ).

\section{miR-185 expression is upregulated by OG}

It was first addressed whether miR-185 expression was regulated in response to different glucose levels. In OG, miR-185 expression significantly increased vs NG

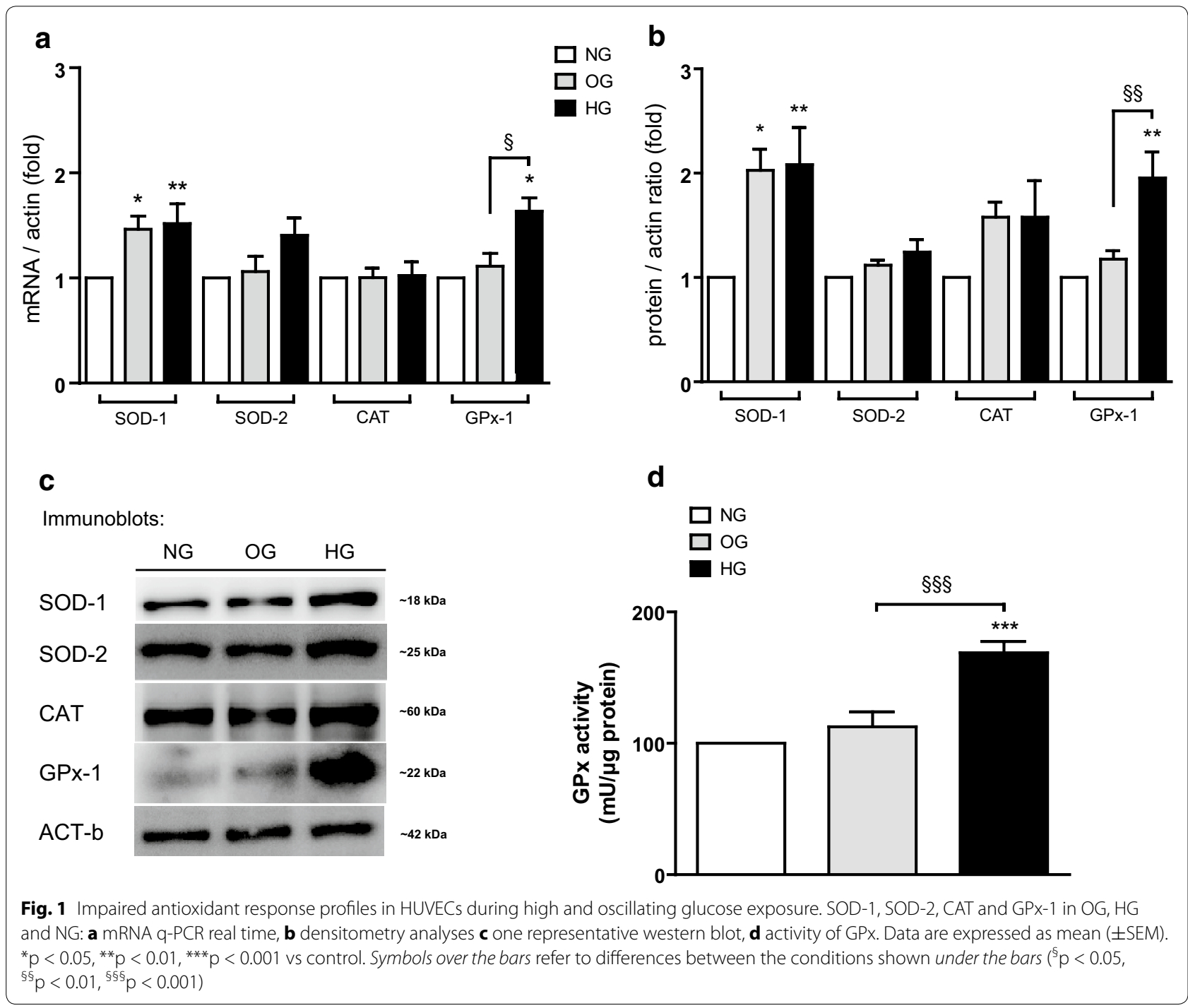


a
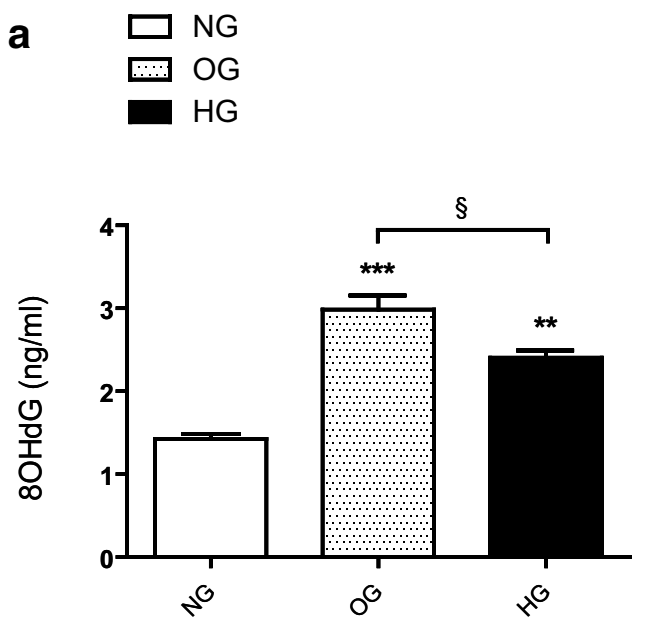
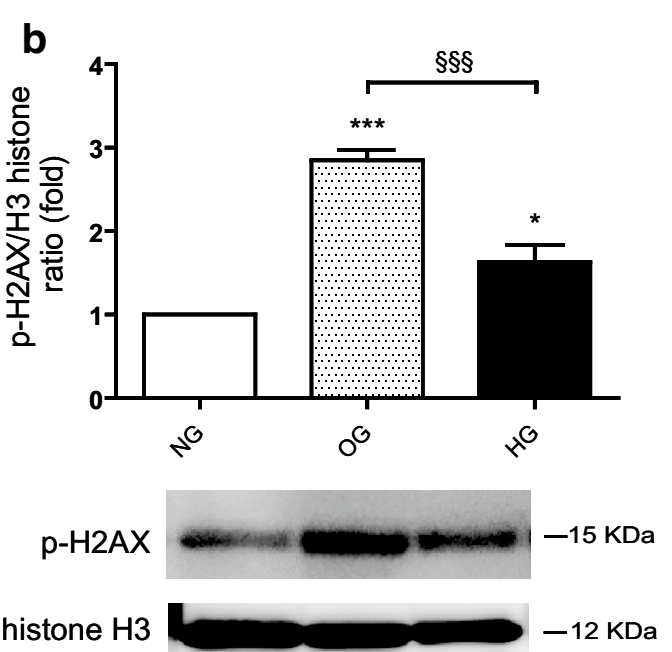

Fig. 2 Effects of oscillating and high glucose on oxidative stress markers in HUVECs. a 8-OHdG content (ng/ml) in HUVECs during OG, HG and NG. b $\mathrm{Y}-\mathrm{H} 2 \mathrm{AX}$ in HUVECs during OG, HG and NG. The results are expressed as mean $( \pm \mathrm{SEM}),\left({ }^{*} p<0.05,{ }^{* *} \mathrm{p}<0.01,{ }^{* * *} \mathrm{p}<0.001\right.$ vs control). Symbols over the bars refer to differences between the conditions shown under the bars $\left({ }^{\S} p<0.05,{ }^{\S \S} p<0.001\right)$

$(\mathrm{p}<0.001)$ and $\mathrm{HG}(\mathrm{p}<0.01)$, while it was unchanged in NG and HG (Fig. 3a).

\section{GPx-1's target prediction}

The short length of the seed region predicts multiple potential target genes in the genome. Acting as the specificity components of ribonucleoprotein silencing complexes, miRNAs pair with target mRNAs at sites complementary to the miRNA $5^{\prime}$ region. Most effective sites map to $3^{\prime}$ untranslated regions ( $3^{\prime}$ UTRs) and pair perfectly with the miRNA seed (nucleotides 2-7), with an additional pair at nucleotide 8 and/or an A across from nucleotide 1 . Using in silico analysis (TargetScanHuman v.5) to predict the target gene of miR-185, we identified two binding sites into $3^{\prime}$-UTR of the GPx-1 gene (Fig. 3b). Although the target prediction was based on poorly conserved site analysis, the mature miR-185 stranded with the seed region in a perfect alignment between nucleotides 2-7 (Fig. 3b). This region is a key predictor of direct binding to the target gene, as suggested by Lewis BP et al. [16]. The ability of an miRNA to translationally repress a target mRNA is largely dictated by the free energy of binding of the first eight nucleotides in the $5^{\prime}$ region of the miRNA. RNA hybrid analysis of GPx-1 $3^{\prime}$-UTR offered further support for direct binding of miR-185 to the site; the predicted binding of miR-185 on GPx-1 3'-UTR had highly favorable predicted minimum free energy scores of $\Delta G$ (Gibbs free energy) $=-23.4 \mathrm{kcal} / \mathrm{mol}$, consistent with known miRNA targeting [17].
miR-185 interacts with GPx-1 3'-UTR

In the present study we confirmed the Targetscan in silico predictions demonstrating for the first time a specific and significant interaction between GPx-1 3'UTR and miR-185 using luciferase assay. Co-transfection of HUVEC with GPx-1 $3^{\prime}$ UTR and miR-185 resulted in a significant down regulation of the luciferase light emission (RLU, Relative Light Unit) compared to cells cotransfected with pMIR alone ( $<0.05)$, and with pMIR plus mimic miR-185 ( $<$ < 0.05) (Fig. 3c). Three independent experiments and two replicated transfections were performed.

\section{Endogenous GPX-1 levels are differentially modulated in OG rather than HG by miR-185}

Because GPx-1 expression, with respect to NG, was unchanged in OG and increased in HG, we explored the effects of miR-185 on GPx-1 target gene. OG-induced upregulation of miR-185 was associated with unchanged levels of GPx-1, as detected by q-PCR and Western blot (Fig. 1a-c). To define the effects of miR-185 on GPx-1 gene regulation, miR-185 expression was silenced using the miR-185 Anti-miR ${ }^{\mathrm{TM}}$ miRNA inhibitor. We transfected $1 \mathrm{nmol} / \mathrm{l}$ of anti-miR-185 in HUVECs at the end of culture in NG, OG and HG (Fig. 4a). The transfection efficiencies, assessed by measuring miR-185 expression in q-PCR (Fig. 4a), were $\geq 80 \%$. No difference in NG compared with scrambled miRNA transfection was found. Our results demonstrated that the intracellular levels of miR-185 in transfected samples (Fig. 4a) were reduced in 


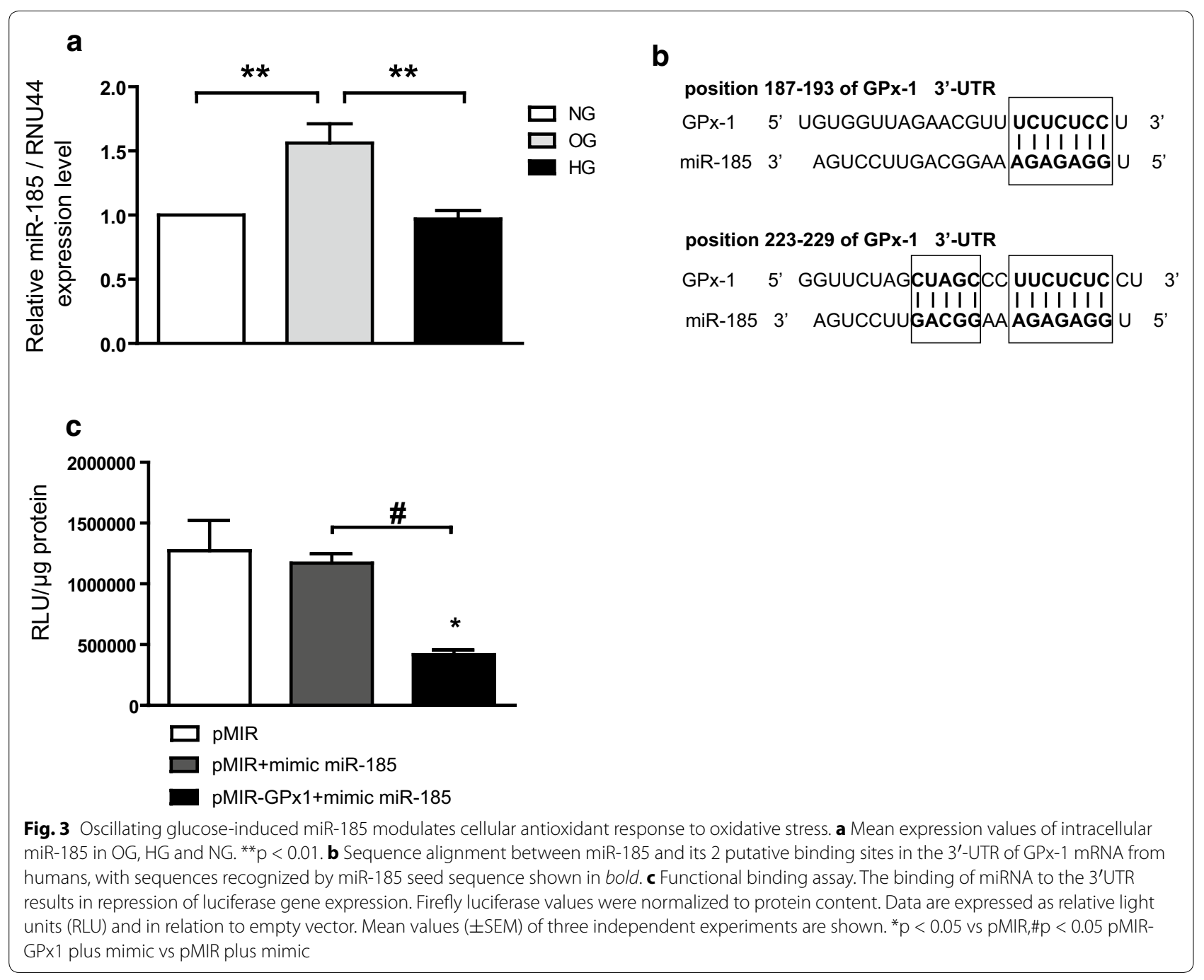

a significant manner (OG vs NG, $\mathrm{p}<0.01$; OG vs HG, $\mathrm{p}<0.001$; Fig. 4a). Trypan blue exclusion dye also demonstrated no cellular loss during transfections (Fig. 4b; Additional file 1:Figure S1). In anti-miR-185 inhibition transfection to knockdown miR-185, we noticed a significant inverted tendency in GPx-1 protein levels (Fig. 4c), suggesting that GPx-1 was modulated specifically by OG and could be a real target for miR-185. We also measured GPx activity during inhibition of miR-185 and found an increased activity in OG in respect to HG ( $p<0.05$, Fig. 4d).

\section{Discussion}

In vitro studies report that OG is more dangerous than HG for several kinds of cells [3-7]. Moreover, these studies consistently prove that this is due to an increase in oxidative stress generation in OG compared to HG [3-7].

The intracellular antioxidant defense system plays a key role in protecting cells during the generation of oxidative stress: generally under these conditions there is an increase in key intracellular antioxidant enzymes, such as SODs, CAT and GPx, aiming to protect cells [8]. This response is also active in oxidative stress generated by HG with the only exception of SOD-2, which does not increase [18].

In this study, for the first time, we report that during OG the response of an antioxidant enzyme, GPx-1, is defective compared to what happens in HG. The absence of an increase of GPx-1 in OG, compared to HG, in our opinion, can convincingly explain the more damaging effects of OG on cells as compared to HG. When GPx-1 does not increase, clearly the oxidative stress produced in cells by glucose is more dangerous to the cells. This hypothesis is further supported by our data regarding the markers of oxidative stress, which are more elevated in OG than in HG.

miRNAs are known as important regulators for target mRNA stability and translation. Recently, it has 

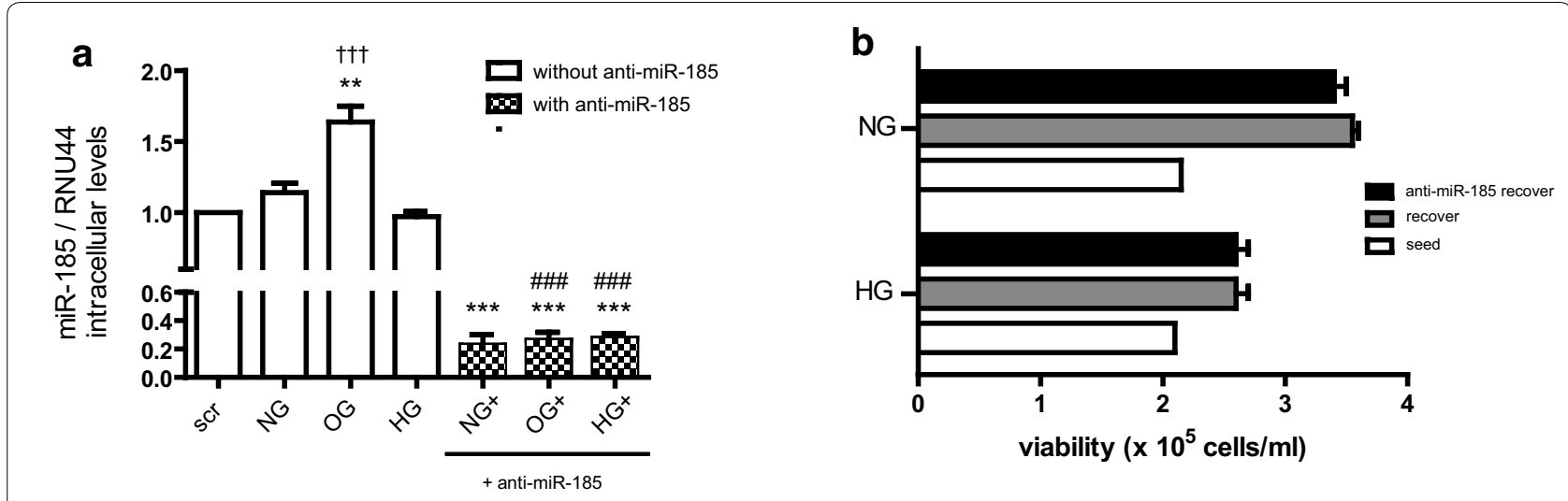

C
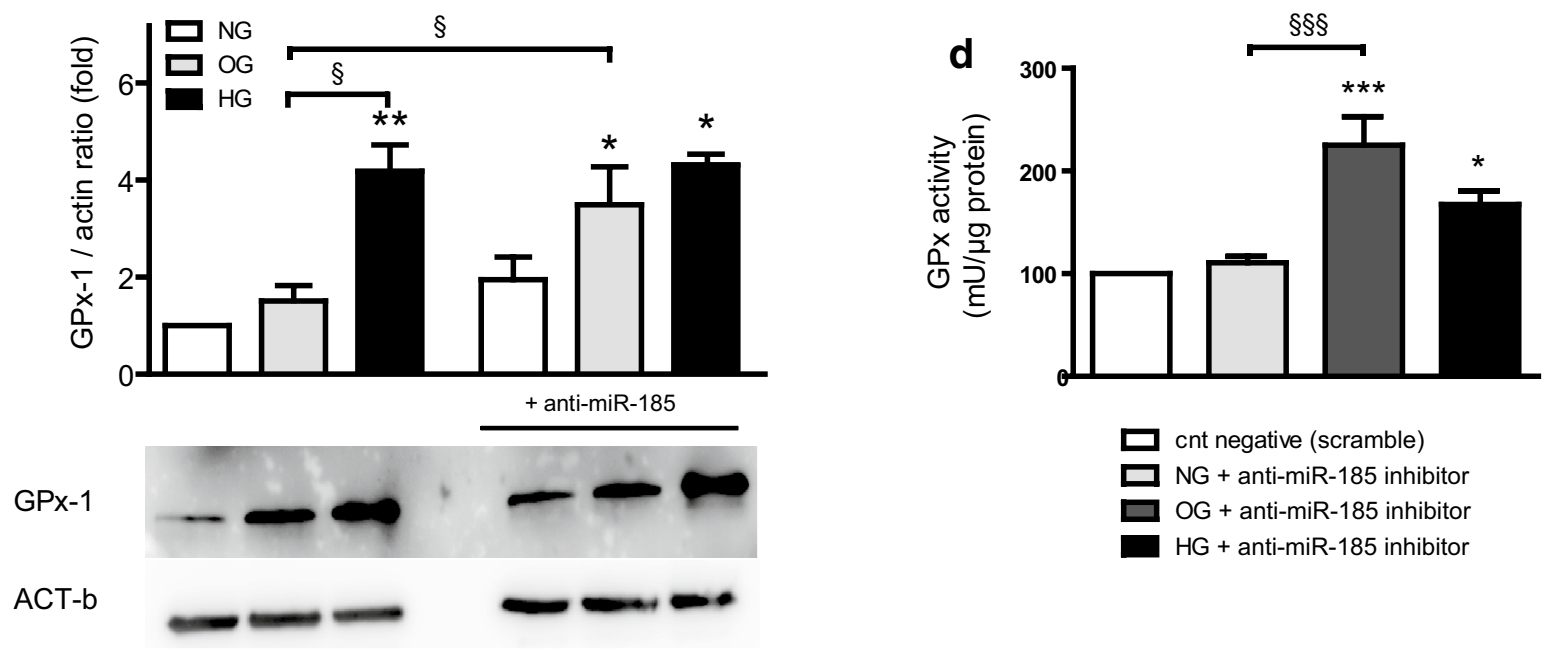

Fig. 4 Knock-down of miR-185 under high and oscillating glucose. a q-PCR of miR-185 in HUVECs cultured in OG, HG and NG, following transfection with inhibitor of miR-185. Data are means ( \pm SEM, ${ }^{* *} p<0.01,{ }^{* * *} p<0.001$ vs control; ${ }^{* \# \#} p<0.001$ vs OG vs OG + , and HG vs HG+; ${ }^{++\dagger} p<0.001$ OG vs HG on the left bars). b Number of viable cells in cell suspensions with anti-miR-185 inhibitor. c Western blot analysis of GPx-1 with or without anti-miR-185 during NG, OG and HG. d GPx activity in HUVECs during miR-185 inhibition in scrambled sample during NG, OG and HG. The results are expressed as mean $( \pm \mathrm{SEM}),{ }^{*} \mathrm{p}<0.05,{ }^{* * *} \mathrm{p}<0.001 \mathrm{vs}$ control). Symbols over the bars refer to differences between the conditions shown under the bars $\left({ }^{\S \S} \mathrm{p}<0.01\right)$

been shown their influence on many cellular functions including glucose metabolism [19]. Important roles for these miRNAs have emerged in the control of metabolic pathways, as suggested by studies implicating miRNAs in the regulation of fat metabolism, adipocyte differentiation, energy homeostasis, and glucose-stimulated insulin secretion [20]. Many studies have identified specific miRNAs expression profiles of diabetes and described the critical roles of miRNAs in insulin secretion [21, 22], pancreatic development and function [22] and diabetic cardiovascular complications [23]. However, the role of several miRNAs, such as miR-21, -146a [24, 25] and let7A [26] as regulators in inflammation and oxidative stresses has been reported.
We have found that OG increases miR-185 expression, a phenomenon that convincingly leads to a decreased expression of its target GPx-1. MIR185 cytogenetic location was found by genomic sequence analysis; Wang et al. (2013) [27] mapped the gene within the first intron of the C22ORF25 gene (also known as Transport and golgi organization 2 homolog, Tango2) on chromosome 22q11.21 in sense orientation. Deletion of this region it has been associated with Di George syndrome, and consequently loss of miR-185 contributes to the cardiac defects in the syndrome [28]. In mouse was detected highest relative miR-185 expression in liver and has been related to lipid metabolism [27]. Recent experimentally validated targets for miR-185, such as Camk2d, Ncx1, 
and Nfatc3 have been related to cardiac diseases $[29,30]$. Moreover, IL-10R $\alpha$ was found a direct target of miR-185, demonstrating a further role in inflammation [31].

Our study suggests a key role of miR-185 in the dangerous effects of OG. If confirmed in vivo, the evidence of a defective GPx-1 response to OG could help explain the mounting evidence linking glucose variability with diabetic complications $[1,2]$. In recent years it has emerged the hypothesis that glucose variability can contribute to the development of complications in diabetes. Recently, high blood glucose variability has been defined as an independent determinant of increased lipid and decreased fibrous contents with larger coronary plaque burden [32] and may impact the formation of lipid-rich plaques and thinning of fibrous cap in CAD patients on lipid-lowering therapy [33]. Moreover, one-year visit-to-visit glucose variability predicted development of end stage renal disease in T2DM patients [34] and was independently associated with the presence of cardiovascular autonomic neuropathy in patients with inadequately controlled T2DM [35]. In our previous study we found that in oscillating and high glucose, total endoglin, its soluble form (sENG), KLF-6 and HIF-1 $\alpha$ were significantly increased [36], and glucose variability reduction via continuous subcutaneous insulin infusion in T1DM increases circulating EPCs levels, suggesting a novel mechanism of vascular damage by oscillating glucose [37].

Consistently, the activity of the antioxidant enzymes CAT, SODs and of GPx has been described as defective in diabetics with complications [38, 39], and an association in vivo has been found between reduced GPx activity and increased risk of cardiovascular complications in diabetes [40].

Glutathione plays a central role in antioxidant defense [41]. Reduced glutathione detoxifies ROS, such as $\mathrm{H}_{2} \mathrm{O}_{2}$, and lipid peroxides, directly or in a GPx-catalyzed mechanism [42]. GPx-1 is an abundant cytoplasmic enzyme specifically involved in the response to peroxyl-radicals and plays an important role in intracellular detoxification. It has been found to be more effective than CAT in removing intracellular peroxides under many different physiological conditions [42, 43]. Moreover, GPx-1 catalyzes the conversion of $\mathrm{H}_{2} \mathrm{O}_{2}$ or organic hydro-peroxides into water, or its corresponding alcohols, using glutathione as a substrate [44], and protects against oxidative and nitrosative stress in blood vessels [45]. $\mathrm{H}_{2} \mathrm{O}_{2}$ forms the toxic oxygen species hydroxyl radical, which is highly reactive and causes lipid peroxidation, and hydroxide anion, which promotes alkaline tissue damage, a process that is offset in part by CAT and GPx-1-dependent reduction to $\mathrm{H}_{2} \mathrm{O}$. A deficiency in GPx-1 would then lead to an increase in ROS. Thus, a defective GPx-1 response in OG can expose the vasculature to potentially important damage.

\section{Conclusion}

Our study shows for the first time that the exposure of endothelial cells to OG produces an impaired antioxidant response, in particular, that the upregulation of miR-185 contributes to GPx-1 inappropriate response in OG. This finding, in our opinion, contributes to explaining why OG is more dangerous that HG, as reported by several studies [3-7], and further supports the hypothesis that OG may be involved in the development of diabetic complications $[1,2]$.

\section{Additional file}

Additional file 1: Fig S1. The cells viability after 5 days of exposures to glucose.

\section{Abbreviations}

8-OHdG: 8-hydroxy-2'-deoxyguanosine; CAT: catalase; EBM-2: endothelial basal medium; GPx: glutathione peroxidases; GPX-1: glutathione peroxidases-1; $\gamma-\mathrm{H} 2 \mathrm{AX}$ : phosphorylated form of $\mathrm{H}_{2} \mathrm{AX} ; \mathrm{H}_{2} \mathrm{O}_{2}$ : hydrogen peroxide; $\mathrm{HG}$ : high glucose; HUVECs: human umbilical vein endothelial cells; microRNAs: miRNAs; miR-185: microRNA-185; MRE: microRNA recognition element; NG: normal glucose; OG: oscillating glucose; ROS: reactive oxygen species; RLU: relative light unit; SOD-1: copper zinc SOD; SOD-2: manganese SOD; $3^{\prime}$ UTRs: un-translated regions.

\section{Authors' contributions}

LLS contributed to: conception and design of the study, acquisition, analysis and interpretation of data wrote manuscript and approved the manuscript submission. MC, VDN, GP, RT, ARB and SG contributed to: acquisition and analysis, interpretation of data, revising the manuscript and approved the manuscript submission. AC contributed to: conception and design of the study, analysis and interpretation of data wrote manuscript and approved the manuscript submission. All authors read and approved the final manuscript.

\section{Author details}

${ }^{1}$ Department of Cardiovascular Research, IRCCS MultiMedica, Milan, MI, Italy. ${ }^{2}$ Insititut d'Investigacions Biomèdiques August Pi i Sunyer (IDIBAPS) and Centro de Investigación Biomedica en Red de Diabetes y Enfermedades Metabólicas Asociadas (CIBERDEM), Barcelona, Spain. ${ }^{3}$ Experimental Models in Clinical Pathology, INRCA-IRCCS National Institute, Ancona, Italy. ${ }^{4}$ Scientific Direction, INRCA, Via S. Margherita, 5, Ancona 60124, Italy.

\section{Acknowledgements}

The authors wish to thank Kimberly Katte of CIBERDEM for editorial assistance with the manuscript. The authors also thank Fondazione Invernizzi (Milan, Italy), for financial support.

\section{Competing interests}

The authors declare that they have no competing interests.

Received: 20 November 2015 Accepted: 21 April 2016

Published online: 30 April 2016

\section{References}

1. Frontoni S, Di Bartolo P, Avogaro A, Bosi E, Paolisso G, Ceriello A. Glucose variability: an emerging target for the treatment of diabetes mellitus. Diabetes Res Clin Pract. 2013;102:86-95.

2. Ceriello A, Kilpatrick ES. Glycemic variability: both sides of the story. Diabetes Care. 2013;36(Suppl 2):S272-5.

3. Quagliaro L, Piconi L, Assaloni R, Martinelli L, Motz E, Ceriello A. Intermittent high glucose enhances apoptosis related to oxidative stress in 
human umbilical vein endothelial cells: the role of protein kinase $C$ and NAD(P)H-oxidase activation. Diabetes. 2003;52:2795-804.

4. Sun J, Xu Y, Sun S, Sun Y, Wang X. Intermittent high glucose enhances cell proliferation and VEGF expression in retinal endothelial cells: the role of mitochondrial reactive oxygen species. Mol Cell Biochem. 2010;343:27-35

5. Sun J, Xu Y, Deng H, Sun S, Dai Z, Sun Y. Intermittent high glucose exacerbates the aberrant production of adiponectin and resistin through mitochondrial superoxide overproduction in adipocytes. J Mol Endocrinol. 2010:44:179-85.

6. Sun LQ, Chen YY, Wang X, Li XJ, Xue B, Qu L, et al. The protective effect of alpha lipoic acid on Schwann cells exposed to constant or intermittent high glucose. Biochem Pharmacol. 2012;84:961-73.

7. Kohnert KD, Freyse EJ, Salzsieder E. Glycaemic variability and pancreatic B-cell dysfunction. Curr Diabetes Rev. 2012;8:345-54.

8. Matés JM, Sánchez-Jiménez F. Antioxidant enzymes and their implications in pathophysiologic processes. Front Biosci. 1999;4:D339-45.

9. Rottiers V, Naar AM. MicroRNAs in metabolism and metabolic disorders. Nat Rev Mol Cell Biol. 2012;13:239-50.

10. Bartel DP. MicroRNAs: target recognition and regulatory functions. Cell. 2009;136:215-33.

11. Kato M, Castro NE, Natarajan R. MicroRNAs: potential mediators and biomarkers of diabetic complications. Free Radic Biol Med. 2013;64:85-94.

12. Kim V, Han J, Siomi MC. Biogenesis of small RNAs in animals. Nat Rev Mol Cell Biol. 2009;10:126-39.

13. Maciel-Dominguez A, Swan D, Ford D, Hesketh J. Selenium alters miRNA profile in an intestinal cell line: evidence that miR-185 regulates expression of GPX2 and SEPSH2. Mol Nutr Food Res. 2013;57(12):2195-205.

14. Grimson A, Farh KK, Johnston WK, Garrett-Engele P, Lim LP, Bartel DP. microRNA targeting specificity in mammals: determinants beyond seed pairing. Mol Cell. 2007;27:91-105.

15. Rehmsmeier M, Steffen P, Hoechsmann M, Giegerich R. Fast and effective prediction of microRNA/target duplexes RNA. RNA. 2004;10:1507-17.

16. Lewis BP, Shih $I H$, Jones-Rhoades MW, Bartel DP, Burge CB. Prediction of mammalian microRNA targets. Cell. 2003;115:787-98.

17. Doench JG, Sharp PA. Specificity of microRNA target selection in translational repression. Genes Dev. 2004;18(5):504-11.

18. Ceriello A, Russo PD, Amstad P, Cerutti P. High glucose induces antioxidant enzymes in human endothelial cells in culture. Evidence linking hyperglycemia and oxidative stress. Diabetes. 1996:45:471-7.

19. Zhu H, Shyh-Chang N, Segrè AV, Shinoda G, Shah SP, Einhorn WS, et al. The Lin28/let-7 axis regulates glucose metabolism. Cell. 2011;147:81-94.

20. Krutzfeldt J, Stoffel M. microRNAs: a new class of regulatory genes affecting metabolism. Cell Metab. 2006:4:9-12

21. Puigserver P, Rodgers JT. Foxa2, a novel transcriptional regulator of insulin sensitivity. Nat Med. 2006;12:38-9.

22. Poy MN, Eliasson L, Krutzfeldt J, Kuwajima S, Ma X, Macdonald PE, et al. A pancreatic islet-specific microRNA regulates insulin secretion. Nature. 2004;432:226-30

23. Shantikumar S, Caporali A, Emanueli C. Role of microRNAs in diabetes and its cardiovascular complications. Cardiovasc Res. 2012;93:583-93.

24. Cheng Y, Liu X, Zhang S, Lin Y, Zhang C. microRNA-21 protects against the $\mathrm{H}(2) \mathrm{O}(2)$-induced injury on cardiac myocytes via its target gene PDCD4. J Mol Cell Cardiol. 2009;47(1):5-14.

25. Wang HJ, Huang YL, Shih YY, Wu HY, Peng CT, Lo WY. MicroRNA-146a decreases high glucose/thrombin-induced endothelial inflammation by inhibiting NADPH oxidase 4 expression. Mediators Inflamm. 2014;2014:379537.

26. Song J, Lee JE. ASK1 modulates expression of microRNA Let7A in microglia under high glucose in vitro condition. Front Cell Neurosci. 2015;9:198.
27. Wang L, Jia X, Jiang H, Du Y, Yang F, Si S, Hing B. MicroRNAs 185 , 96, and 223 repress selective high-density lipoprotein cholesterol uptake through posttranscriptional inhibition. Mol Cell Biol. 2013;33-10:1956-64.

28. de la Morena MT, Eitson JL, Dozmorov IM, Belkaya S, Hoover AR, Anguiano E, Pascual MV, van Oers NS. Signature microRNA expression patterns identified in humans with 22q11.2 deletion/DiGeorge syndrome. Clin Immunol. 2013;147(1):11-22.

29. Kim JO, Song DW, Kwon EJ, Hong SE, Song HK, Min CK, Kim do H. miR185 plays an anti-hypertrophic role in the heart via multiple targets in the calcium-signaling pathways. PLoS One. 2015;10(3):e0122509.

30. Kim JO, Song DW, Kwon EJ, Hong SE, Song HK, Min CK, Kim do H. miR185 plays an anti-hypertrophic role in the heart via multiple targets in the calcium-signaling pathways. PLoS One. 2015;10(3):e0122509.

31. Venza I, Visalli M, Beninati C, Benfatto S, Teti D, Venza M. IL-10Ra expression is post-transcriptionally regulated by miR-15a, miR-185, and miR-21 in melanoma. BMC Med Genomics. 2015;8:81.

32. Okada $\mathrm{K}$, et al. Association between blood glucose variability and coronary plaque instability in patients with acute coronary syndromes. Cardiovasc Diabetol. 2015;14:111.

33. Kuroda $\mathrm{M}$, et al. Association between daily glucose fluctuation and coronary plaque properties in patients receiving adequate lipid-lowering therapy assessed by continuous glucose monitoring and optical coherence tomography. Cardiovasc Diabetol. 2015;14:78.

34. Yang YF, et al. Visit-to-visit glucose variability predicts the development of end-stage renal disease in type 2 diabetes: 10-year follow-up of Taiwan diabetes study. Medicine. 2015;94(44):e1804.

35. Jun JE, et al. The association between glycemic variability and diabetic cardiovascular autonomic neuropathy in patients with type 2 diabetes. Cardiovasc Diabetol. 2015:14:70.

36. La Sala L, et al. Oscillating glucose and constant high glucose induce endoglin expression in endothelial cells: the role of oxidative stress. Acta Diabetol. 2015;52:505-12.

37. Maiorino Ml, et al. Reducing glucose variability with continuous subcutaneous insulin infusion increases endothelial progenitor cells in type 1 diabetes: an observational study. Endocrine. 2016;52(2):244-52.

38. Ceriello A, Morocutti A, Mercuri F, Quagliaro L, Moro M, Damante G, et al. Defective intracellular antioxidant enzyme production in type 1 diabetic patients with nephropathy. Diabetes. 2000;49:2170-7.

39. Hodgkinson AD, Bartlett T, Oates PJ, Millward BA, Demaine AG. The response of antioxidant genes to hyperglycemia is abnormal in patients with type 1 diabetes and diabetic nephropathy. Diabetes. 2003:52:846-51

40. Hamanishi T, Furuta H, Kato H, Doi A, Tamai M, Shimomura H, et al. Functional variants in the glutathione peroxidase-1 (GPx-1) gene are associated with increased intima-media thickness of carotid arteries and risk of macrovascular diseases in japanese type 2 diabetic patients. Diabetes. 2004;53:2455-60.

41. Kalinina EV, Chernov NN, Novichkova MD. Role of glutathione, glutathione transferase, and glutaredoxin in regulation of redox-dependent processes. Biochemistry. 2014;79:1562-83.

42. Antunes F, Han D, Cadenas E. Relative contributions of heart mitochondria glutathione peroxidase and catalase to $\mathrm{H}(2) \mathrm{O}(2)$ detoxification in in vivo conditions. Free Radic Biol Med. 2002;33:260-7.

43. Jones DP. Intracellular inhibition of catalase by al pha-methyldopa. Res Commun Chem Pathol Pharmacol. 1981:33:215-22.

44. Lei XG, Cheng WH, McClung JP. Metabolic regulation and function of glutathione peroxidase-1. Annu Rev Nut. 2007:27:41-61.

45. Forgione MA, Weiss N, Heydrick S, Cap A, Klings ES, Bierl C, et al. Cellular glutathione peroxidase deficiency and endothelial dysfunction. Am J Physiol Heart Circ Physiol. 2002;282:H1255-61. 